\title{
Aggrecan Chondroitin Sulfate Epitope 846 Measurement
}

National Cancer Institute

\section{Source}

National Cancer Institute. Aggrecan Chondroitin Sulfate Epitope 846 Measurement. NCI

Thesaurus. Code C112220.

The determination of the amount of aggrecan chondroitin sulfate epitope 846 present in a sample. 